\title{
Prevalence of Abomasal Nematodes in Sheep Slaughtered at Baneh Town
}

\author{
${ }^{1}$ Garedaghi Yagoob, ${ }^{1}$ Hashemzadefarhang Hossein and ${ }^{2}$ Fattahi Asso \\ ${ }^{1}$ Department of Pathobiology, Tabriz Branch, Islamic Azad University, Tabriz, Iran \\ ${ }^{2}$ Department of Veterinary Medicine, Tabriz Branch, Islamic Azad University, Tabriz, Iran
}

Received 2012-09-12, Revised 2012-12-10; Accepted 2013-09-09

\begin{abstract}
Livestock production covers up to $40 \%$ of the gross value of agricultural production globally. Gastrointestinal nematodes of small ruminants are one of the major causes of productivity loss. This study was carried out to determine the correlation between the prevalence, seasonal incidence and geographical distribution of abomasal worm infection of native sheep in Baneh Town of Iran, suitable for animal husbandry. From February 2011 to February 2012 the contents of abomasums of 400 sheep were washed separately in a 100 mesh sieve. The worms present in each abomasum were collected separately, counted and preserved in $70 \%$ alcohol containing $5 \%$ glycerin for identification to the species. The overall percentage of infection was $25.36 \%$ and Haemonchus contortus, Teladorsagia circumcincta, Marshallagia marshalli, Ostertagia occidentalis, Ostertagia trifurcata and Parabronema skrjabini were 6 species identified in studied areas. The overall prevalence rate and intensity of worm's burden as representative of Iran, were low, although Teladorsagia circumcincta was the most prevalent and frequent worm species found. Using Chi-Square and ANOVA, no significant relationship was found between prevalence, season, age and sex.
\end{abstract}

Keywords: Prevalence, Abomasal Nematodes, Sheep, Baneh Town, Iran

\section{INTRODUCTION}

Gastro-intestinal nematode parasite infections are a major constraint to the sheep industry and cause production losses, increased costs of management and treatment and even mortality in severe cases. Abomasum is one of the most important sites for living bursate nematodes belonging to Trichostrongylidae family in small ruminants, because it is the site location for 3 pathogen species of GI nematodes e.g., Haemonchus spp. Teladorsagia spp. Ostertagia spp. and Trichostrongylus spp. Meanwhile it was shown that gastrointestinal nematodes could be harmful to the health of infected animals and causes economic losses due to mortalities, reduce weight gain and other production losses (Skerman et al., 1967; Uriarte et al., 2003; Vlassoff and Mckenna, 2010; Sissay et al., 2007). On the other hand the development of parasites including gastrointestinal nematodes of ruminants is entirely weather dependant
(Sissay et al., 2007; Tariq et al., 2008). Although the effects of helminth infections on production of particular livestock species depend mostly upon the age of the animal, breed, parasite species involved and the time at which infections with infective larvae begin, the intensity of the worm population (Al-Shaibani et al., 2008). Seasonal incidence of GI nematodiasis is done to find rise to a peak and decline so that the treatment can be timed to prevent development of serious infection and to reduce contamination of pastures with eggs and larvae. If major differences occur in climatic condition of different zones, epidemiological studies must be carried out according to each zone.

In several studies big differences have been shown in the prevalence and intensity of small ruminant's GI nematodes according to different climatic condition (Skerman et al., 1967; Uriarte et al., 2003; Tariq et al., 2008; El-Azazy, 1995). Fifty two millions sheep are scattered in different regions of Iran, (Kamalzadeh et al., Corresponding Author: Garedaghi Yagoob, Department of Pathobiology, Tabriz Branch, Islamic Azad University, Tabriz, Iran 
2008), where climatologically it is divided into 4 regions (Skerman et al., 1967). The majorities of sheep population in Iran are grazing in the pasture and are in permanent contact with pasture harboring 3rd stage larvae of GI nematode.

In a comprehensive study in 2 zones (Zone 1 and 2), which carried out by Skerman et al. (1967), epidemiology, seasonal incidence and economic importance of gastrointestinal nematode of small ruminants of Iran was studied. Since then, in several studies, no attention has been paid to the effects of climatic conditions of different zones on the epidemiology of GI nematodes. This study was carried out to have new understanding of abomasal helminthiasis in baneh Town of Iran.

\section{MATERIALS AND METHODS}

\subsection{Experimental Animals and Parasitological Techniques}

From February 2011 to February 2012, the age and sex of 400 native sheep from baneh town were determined and their abomasums were collected. The contents of each abomasums were washed under running water using a 100 mesh sieve and the present nematodes were counted and preserved in $70 \%$ alcohol containing $5 \%$ glycerin. Because the intensities of nematode infections were low, hundred percent of nematodes were subjected to microscopical examination and they were identified according to morphological characteristics described by Skrjabin and Shikhobalova (1954).

\subsection{Data Analysis}

Prevalence of parasite species was calculated as number of individuals of a host species infected with a particular parasite species/number of host examined. Chi-square test was employed to examine the effect of region, season, age and sex of the host on the level of parasitism. ANOVA test was used to show the correlation between mean worm burden, season, age and sex of the host. In all the analyses, $p=0.05$ was for significance.

\section{RESULTS AND DISCUSSION}

The prevalence and intensity of nematode infections in which could be taken as representative of infection for whole country are summarized in Table 1. Six species of nematodes with low prevalence and intensity were found in examined abomasums.
Table 1. The total prevalence and intensity of abomasal nematode infection in Baneh town, Iran

\begin{tabular}{|c|c|c|c|}
\hline Parasite & $\begin{array}{l}\% \\
\text { Prevalence }\end{array}$ & $\begin{array}{l}\text { Mean number of } \\
\text { worm per infected } \\
\text { abomasum }\end{array}$ & $\begin{array}{l}\text { Maximum } \\
\text { burden }\end{array}$ \\
\hline Haemonchus contortus & 2.10 & 11.5 & 32 \\
\hline Parabronema skrjabini & 4.30 & 9.8 & 18 \\
\hline Marshallagia marshalli & 10.11 & 81.3 & 410 \\
\hline Teladorsagia circumcincta & 17.31 & 102.1 & 523 \\
\hline Ostertagia trifurcata & 0.06 & 6.8 & 22 \\
\hline Ostertagia occidentalis & 2.71 & 7.1 & 17 \\
\hline
\end{tabular}

The overall prevalence and intensity was $25.36 \%$ and 98.5 respectively. Among the species found Teladorsagia circumcincta was the most prevalent and frequent species. No significant correlation was observed between the prevalence of infection with seasons, ages, sexes.

\section{CONCLUSION}

The results of present study revealed that as the weight of the animal increases the parasitic infection decreases. This might be due to the development of acquired immunity. The interpretation of our results, enlighten a new concept on the epidemiology of abomasal helminths of sheep from Iran and show a reduction in the number of species recorded, prevalence rate and intensity of infection and the absence of seasonality. As far as the prevalence and intensity of abomasal worm infections are concerned, our findings are in consonant with studies carried out in this field in recent years (Oliaee et al., 2008; Moghaddar and Afrahi, 2008; Ranjbar-Bahadori et al., 2007) but not with old reports. In the survey, carried out two decades ago, (Eslami and Nabavi, 1976), dramatic differences and decrease occurs with our results in the prevalence and mean intensity of 3 common nematode species; e.g., H.contortus, T.circumcincta and M. marshalli. Although as it is notices by Suntin-Duran et al. (2008), worm burden may have been underestimated in our study, as we did not also search or recover fourth stage larvae that may have been presented as the arrested population in sheep, a phenomenon recorded for M. marshalli, H.contortus, N. filicollis and T. colubriformis in sheep from Fars province, southern part of Iran (Michel and Hooshmand-Rad, 1978). A striking founding is the absence of 3 species of Trichostrongylus spp in the present research, which were recorded from sheep with high prevalence and mean intensity range (17-58\% and 8-740 respectively), (Eslami and Nabavi, 1976). This shows the more responsiveness of these species to 
strategic anthelmintic treatment starting two decades ago. On the other hand, our findings uniformity of seasonality and prevalence inbaneh town, are in contrast with Skerman et al. (1967). These remarkable changes are firstly related to the governmental strategic treatment of Iranian sheep and goats' population since 1968 twice a year for a period of 10 years, with albendazole and its continuation later on by sheep owner themselves. Secondly to periodical draught encountered in Iran in last two decades. Meanwhile the economic benefits of strategic treatment and raise the price of meat bring the sheep owner to the assumption that more treatment will lead to more meat, the result of which, among other things, was production of resistance of some trichostrongylids to albendazole and tetramisole. Moreover limitation in grazing pastures due to rise in price of land decreased the available pastures and its compensation by hand feeding and hence less worm infections.

According to several studies carried out in Iran, (Skerman et al., 1967; Nabavi et al., 2011) and other parts of the world, (Sissay et al., 2007; Taylor et al., 2013; Vlassoff et al., 2001) gastrointestinal nematodes could be harmful to the health of infected animals and causes economic losses due to mortalities, reduce weight gain and other production losses. As far as the site location of GI nematodes are concern, abomasums has a strategic situation, because 3 important pathogenic nematodes of GI e.g., H. contortus, Ostertagia spp. and Trichostrongylus spp. lives in this organ. It seems likely even to take into consideration the mean number of multispecies nematodes infections reported in this study (98.5) no pathogenecity can be attributed to them. As far as abomasal helminths fauna of sheep is concern, it seems likely that the similar species are prevalent in countries neighboring Iran such as Turkey (Umur and Yukari, 2005), Iraq (Kadhim, 1972; Altaif and Issa, 1983), to a lesser extent in Pakistan (Al-Shaibani et al., 2008), where $H$. contortus was the most prevalent species and Ethiopia a country with completely different climatic conditions, where $T$. circumcinct, $H$. contortus and Trichostrongylus axei were the prevalent species (Abunna et al., 2009).

The results of this study show a harmony between fauna, prevalence and even intensity of abomasal helminths of sheep. For actual abomasal worm infections and its extent to GI nematodiasis, no chemotherapy is recommended, but it is necessary to prevent emerging sever and new resistance of GI helmiths to anthelmintic compounds and reemerging a new population of helminths harmful to the health and economy of sheep breeding by monitor the trend of infection in the future. The differences in prevalence reported by these studies could be accounted on the basis of differential management practices (Garedaghi et al., 2011).

\section{ACKNOWLEDGMENT}

The researchers wish to thanks the Islamic Azad University, Tabriz Branch, Tabriz, Iran for the financial supports and all laboratory technicians for technical aids in this project.

\section{REFERENCES}

Abunna, F., B. Kumsa, B. Megersa, A. Regassa and E. Debela, 2009. Abomasal nematodes: Prevalence in small ruminants slaughtered at Bishooftu town. Ethiopia. J. Vet. Med., 7: 1-6.

Al-Shaibani, I.R.M., M.S. Phulan, A. Arijo and T.A. Qureshi, 2008. Epidemiology of ovine gastrointestinal nematodes in Hyderabad district, Pakistan. Pak. Vet. J., 28: 125-130.

Altaif, K.I. and W.H. Issa, 1983. Epidemiology of gastrointestinal parasites of Awassi sheep in Iraq. Vet. Parasitol., 12: 51-58. DOI: 10.1016/03044017(83)90087-0

El-Azazy, O.M.E., 1995. Seasonal changes and inhibited development of the abomasal nematodes of sheep and goats in Saudi Arabia. Vet. Parasitol., 58: 91-98. DOI: 10.1016/0304-4017(94)00696-A

Eslami, A. and L. Nabavi, 1976. Species of gastrointestinal nematodes of sheep from Iran. Bull. Soc. Pathol. Exotique, 69: 92-95.

Garedaghi, Y., A.P. Rezaii Saber and R. Attaremadraki, 2011. Efficacy of closantel 5\% against cattle gastrointestinal parasites. Am. J. Anim. Vet. Sci., 6: 112-116. DOI: 10.3844/ajavsp.2011.112.116

Kadhim, J.K., 1972. A survey of gastrointestinal helminth parasites of sheep in Iraq. Tropical Anim. Health Product., 4: 100-112. DOI: 10.1007/BF02359744

Kamalzadeh, A., M. Rajabbaigy and A. Kiasat, 2008. Livestock production systems and trends in livestock Industry in Iran. J. Agric. Soc. Sci., 4: 183188.

Michel, J.F. and P. Hooshmand-Rad, 1978. Arrested development of nematodes in sheep in southern Iran. Parasitology, 77: 18.

Moghaddar, N. and A. Afrahi, 2008. Gastrointestinal helminthosis in sheep in Iran. J. Vet. Med., 22: 25-28. 
Nabavi, R., A. Eslami, H.R. Shokrani, S. Bokaie and P. Shayan et al., 2011. Study on the prevalence, intensity, seasonal dynamics of Abomasal Helminths in sheep from different climatic zones of Iran. World Applied Sci. J., 12: 441-445.

Oliaee, A., A. Eslami, S. Bokaie and N. HoghooghiRad, 2008. Study on the prevalence and seasonal incidence of abomasal nematodes of Kazeroon ruminants. Islamic Azad University, Garmsar Branch, Garmsar, Iran.

Ranjbar-Bahadori, S.H., A. Eslami and R.A.E. Samani, 2007. Study on the helminth infection of native ruminants of Golestan province. Iranian J. Vet. Res., 63: 303-305.

Sissay, M., A. Uggla and P. Waller, 2007. Epidemiology and seasonal dynamics of gastrointestinal nematode infections of sheep in a semi-arid region of eastern Ethiopia. Vet. Parasitol., 143: 311-321. DOI: 10.1016/j.vetpar.2006.08.026

Skerman, K.O., A.A. Shahlapoor, A. Eslami and M. Eliazian, 1967. Observation on the incidence, epidemiology, control and economic importance of gastro-intestinal parasites of Sheep and Goats in Iran. Vet. Med. Rev.

Skrjabin, K.I. and N.P. Shikhobalova, 1954. Trichostrongylids of Animals and Man. 1st Edn., Academy of Sciences, pp: 704.

Suntin-Duran, M., J.M. Alunda, E.P. Hoberg and C. De la Fuente, 2008. Age distribution and seasonal dynamics of abomasal helminths in wild red deer from Central Spain, J. Parasitol., 95: 1031-1037. DOI: $10.1645 / \mathrm{GE}-1109.1$
Tariq, K.A., M.Z. Chishti, F. Ahmad and A.S. Shawl, 2008. Epidemiology of gastrointestinal nematodes of sheep managed under traditional husbandry system in Kashmir valley. Vet. Parasitol., 158: 138143. DOI: 0.1016/j.vetpar.2008.06.013

Taylor, M.A., R.L. Coop and R.L. Wall, 2013. Veterinary Parasitology. 3rd Edn., John Wiley and Sons, Chicester, ISBN-10: 1118687116, pp: 600.

Umur, S. and A.B. Yukari, 2005. Seasonal Activity of Gastro-Intestinal Nematodes in Goats in Burdur Region, Turkey. Turkish J. Vet. Anim. Sci., 29: 441-448.

Uriarte, J., M.M. Liorente and J. Valderrabano, 2003. Seasonal changes of gastrointestinal nematode burden in sheep under an intensive grazing system. Vet. Parasitol., 118: 79-92. DOI: 10.1016/j.vetpar.2003.07.030

Vlassoff, A. and P.B. Mckenna, 2010. Nematode parasites of economic importance in sheep in New Zealand. New Zealand J. Zool., 21: 1-8. DOI: 10.1080/03014223.1994.9517971

Vlassoff, A., D.M. Leathwick and A.C.G. Heath, 2001. The epidemiology of nematode infections of sheep. New Zeland Vet. J., 49: 213-221. DOI: 10.1080/00480169.2001.36235 